\title{
Central stars of planetary nebulae: The white dwarf connection
}

\author{
Klaus Werner \\ Institute for Astronomy and Astrophysics, Kepler Center for Astro and Particle Physics, \\ Eberhard Karls University, Sand 1, 72076 Tübingen, Germany \\ email: werner@astro.uni-tuebingen.de
}

\begin{abstract}
This paper is focused on the transition phase between central stars and white dwarfs, i.e. objects in the effective temperature range $100000-200000 \mathrm{~K}$. We confine our review to hydrogen-deficient stars because the common H-rich objects are subject of the paper by Ziegler et al. in these proceedings. We address the claimed iron-deficiency in PG1159 stars and [WC] central stars. The discovery of new Ne VII and Ne VIII lines in PG1159 stars suggests that the identification of $\mathrm{O}$ VII and $\mathrm{O}$ VIII lines that are used for spectral classification of [WCE] stars is wrong. We then present evidence for two distinct post-AGB evolutionary sequences for $\mathrm{H}-$ deficient stars based on abundance analyses of the He-dominated $\mathrm{O}(\mathrm{He})$ stars and the hot DO white dwarf KPD 0005+5106. Finally, we report on evidence for an H-deficient post-super AGB evolution sequence represented by the hottest known, carbon/oxygen-atmosphere white dwarf H 1504+65 and the recently discovered carbon-atmosphere "hot DQ" white dwarfs.
\end{abstract}

Keywords. stars: abundances, stars: AGB and post-AGB, stars: atmospheres, stars: early-type, stars: evolution, white dwarfs, planetary nebulae: general, ultraviolet: stars

\section{Introduction}

Méndez et al. (1986) have introduced the $\mathrm{O}(\mathrm{He})$ and $\mathrm{O}(\mathrm{C})$ designations (besides others) to classify the optical spectra of hot hydrogen-deficient central stars. The $\mathrm{O}(\mathrm{He})$ stars have almost pure He II absorption line spectra whereas the $\mathrm{O}(\mathrm{C})$ stars additionally show strong lines from $\mathrm{C}$ IV and sometimes $\mathrm{O}$ vi. While the $\mathrm{O}(\mathrm{He})$ designation is still in use, the $\mathrm{O}(\mathrm{C})$ stars are today more commonly called PG1159 stars after their prototype PG1159-035. They also comprise some of the stars that were previously termed "O vI" central stars. PG1159 stars are thought to be the progeny of the Wolf-Rayet type central stars (spectral type [WC]), while future evolution will turn them into non-DA white dwarfs. In fact, the picture is a bit more complicated, because a few PG1159 stars show traces of $\mathrm{H}$ and these should become DA white dwarfs. The existence of remnant hydrogen can be explained by a special variant of the late He-shell flash (or born-again star) scenario, which is invoked to be the origin of the H-deficient chemistry.

PG1159 and [WC] stars are particularly useful to test AGB star nucleosynthesis models, because the late He-shell flash that the stars have suffered laid bare the intershell matter between the $\mathrm{H}$ and He burning shells. The observed surface abundances can be directly compared to model predictions for the chemical composition of the AGB star intershell region, where nucleosynthesis of heavy elements proceeds. In this context, one particular problem is the unexpectedly strong iron deficiency claimed for PG1159 and [WC] stars. This is addressed in Sect.2.1. We will also highlight some results on abundance determinations of other metals in PG1159 stars (Sect.2.2). During the course of neon line identifications and abundance determinations it was discovered that the identification of ultrahigh-ionization spectral lines of oxygen - that are commonly used to classify early-type [WC] stars - is wrong (Sect. 2.3). 


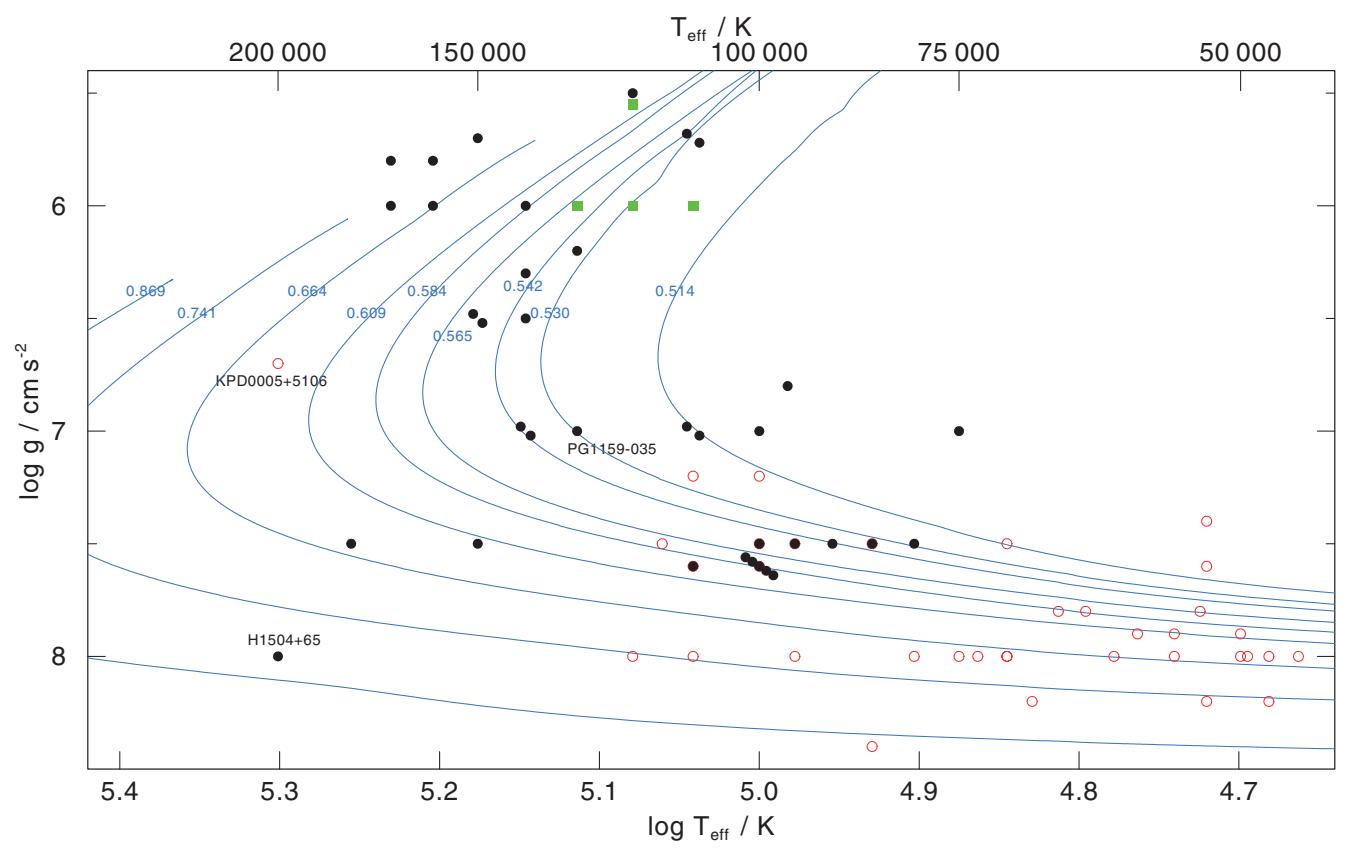

Figure 1. Location of PG1159 stars (filled circles), DO white dwarfs (open circles), and $\mathrm{O}(\mathrm{He})$ stars (squares) in the g- $T_{\text {eff }}$ diagram. Atmospheric parameters for PG1159s and DOs were taken from compilations in Werner \& Herwig (2006) and Althaus et al. (2009), respectively, with some improvements according to more recent work. $\mathrm{O}(\mathrm{He})$ parameters are from Reindl et al. (these proceedings). Evolutionary tracks for non-DA white dwarfs are from Althaus et al. (2009) and they are labeled with the WD mass in $\mathrm{M}_{\odot}$.

While we think that the $\mathrm{O}(\mathrm{C})$ (thus PG1159) stars are part of the sequence $[\mathrm{WC}] \longrightarrow$ PG1159 $\longrightarrow$ non-DA WD, the evolutionary context of $\mathrm{O}(\mathrm{He})$ stars is less clear. They are not explained by the late He-shell flash scenario and there is additional evidence that they indeed belong to a distinct H-deficient post-AGB sequence (see Sect. 3).

Finally, in Sect. 4 we dwell on rather exotic white dwarfs with atmospheres devoid of $\mathrm{H}$ and He, which might be the progeny of super-post AGB stars, i.e. red giants that burned carbon.

\section{PG1159 and [WC] stars}

Recent comprehensive reviews on stellar and atmospheric parameters of [WC] and PG1159 stars can be found in Crowther (2008) and Werner et al. (2008), respectively. The stellar evolution context was summarized by Werner \& Herwig (2006).

In the HRD, [WC] stars are evolving along the constant-luminosity, horizontal part of post-AGB tracks towards high effective temperature. They cover the range of $T_{\text {eff }} \approx 20000 \mathrm{~K}-150000 \mathrm{~K}$. Along the way, the stars shrink such that their surface gravity increases from roughly $\log g=3$ to 6 . With decreasing mass-loss rate, the stars turn into PG1159 stars. The latter populate the region where the stars evolve around the "knee" of the tracks, reaching the maximum effective temperature and subsequently cooling along the WD sequence. Their gravity increases from $\log g=5.5$ to 8 . The hottest PG1159 stars have $T_{\text {eff }}$ near $200000 \mathrm{~K}$, while the most evolved ones are observed at about $T_{\text {eff }}=75000 \mathrm{~K}$. At this region, the stars turn into He-rich white dwarfs (or H-rich in the case some trace $\mathrm{H}$ was left) because of gravitational settling of heavy elements. Figure 1 

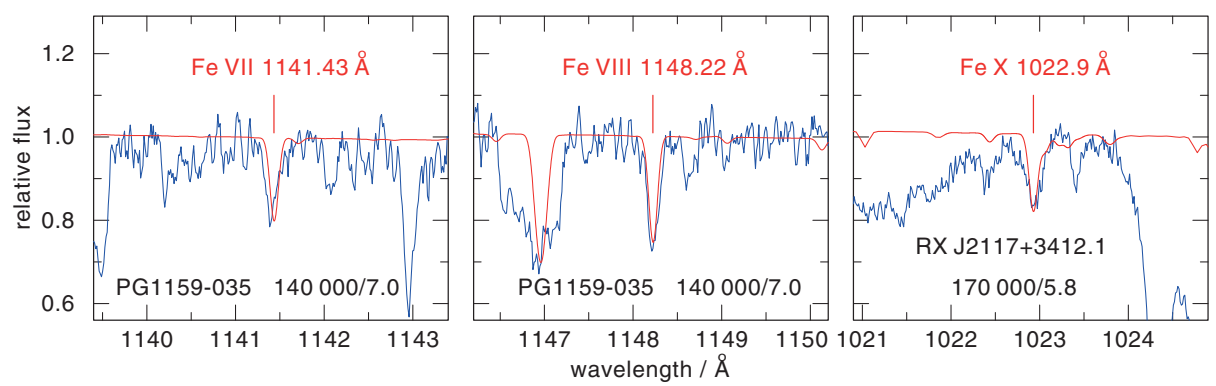

Figure 2. High-ionization iron lines in PG1159 stars. Left two panels: Fe VII and Fe VIII lines in the PG1159 prototype. Right: Fe X line in the central star RX J2117+3412.1. Numbers in panels denote $T_{\text {eff }}$ and $\log g$ of the overplotted model profiles. The Fe abundance in the models is solar.

shows the position of all analyzed PG1159 stars, $\mathrm{O}(\mathrm{He})$ stars, and hot non-DA white dwarfs (spectral type DO) in the $\mathrm{g}-T_{\text {eff }}$ diagram. Note that roughly every other PG1159 star has no associated PN, probably because of its advanced evolutionary state.

The most abundant elements in [WC] and PG1159 atmospheres are He, C, and O. Their relative abundance varies strongly from star to star. For example, a typical mass ratio is displayed by the PG1159 prototype: $\mathrm{He} / \mathrm{C} / \mathrm{O}=33 / 48 / 17$.

As already mentioned in the introduction, abundances of other metals are interesting to compare with nucleosynthesis models. In the next subsections, we concentrate on some specific highlights and problems, focusing on PG1159 stars.

\subsection{Are PG1159 and [WC] stars iron deficient?}

Based on the non-detection of iron lines in any PG1159 star, an iron deficiency up to about one dex was claimed for a number of objects (e.g. Jahn et al. 2007). This is unexplained by stellar models, because they predict an only marginal Fe reduction by $10 \%$ due to neutron captures. An unexpectedly strong Fe depletion was also reported for [WC] stars (e.g. Crowther et al. 1998).

For the PG1159 stars this problem could be resolved recently by the discovery of Fe VIII and Fex lines (Werner et al. 2010, 2011; Fig. 2) in eight of the hottest objects. The derived iron abundances are solar, being in line with stellar models within the analysis error limits. The solar iron abundance in one particular object (PG 1424+535) is consistent with its solar argon abundance (Werner et al. 2007). Argon is an independent metalicity indicator because its abundance remains unchanged in AGB star nucleosynthesis (see, e.g, Lugaro in these proceedings).

In the case of [WC] stars the problem remains. In a first attempt to model Fe VIII and Fex lines in a [WC], Keller et al. (2011 and these proceedings) arrive at a significant iron deficiency (down to 0.3 solar) for the [WCE] NGC 6905. All the more astonishing is their concurrent result of a ten times solar argon abundance.

We note that the newly discovered Fe VIII lines are also present in many H-rich central stars and DO white dwarfs (Werner et al. 2011) and together with Fe viI lines they serve as a new sensitive temperature diagnostics (Ziegler et al. and Mahsereci et al. in these proceedings).

\subsection{Other trace metals in PG1159 stars: N, F, Ne, Si, P, S, Ar}

Far-UV spectroscopy with FUSE augmented by UV spectroscopy with HST was seminal for first-time detection of particular elements and ionization stages in PG1159 stars. Besides iron (Fe viI, Fe viII, Fex), the elements fluorine (F v, F vi; left panel of Fig. 3), phosphorus (P V), neon (Ne VI-VIII, Fig. 4), and argon (Ar VII; right panel of Fig. 3) were 

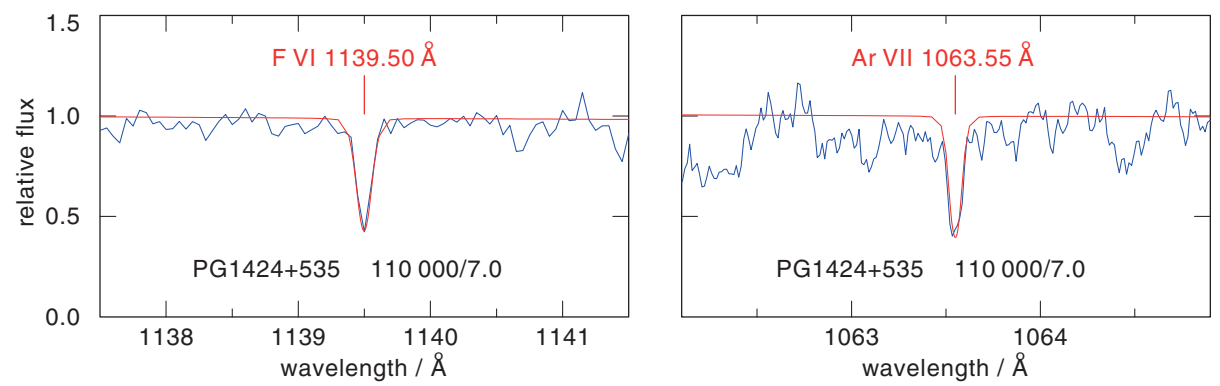

Figure 3. Fluorine line (left panel) and argon line (right panel) in the PG1159 star PG1424+535. The fluorine and argon abundances in the overplotted model are 200 times solar and solar, respectively.
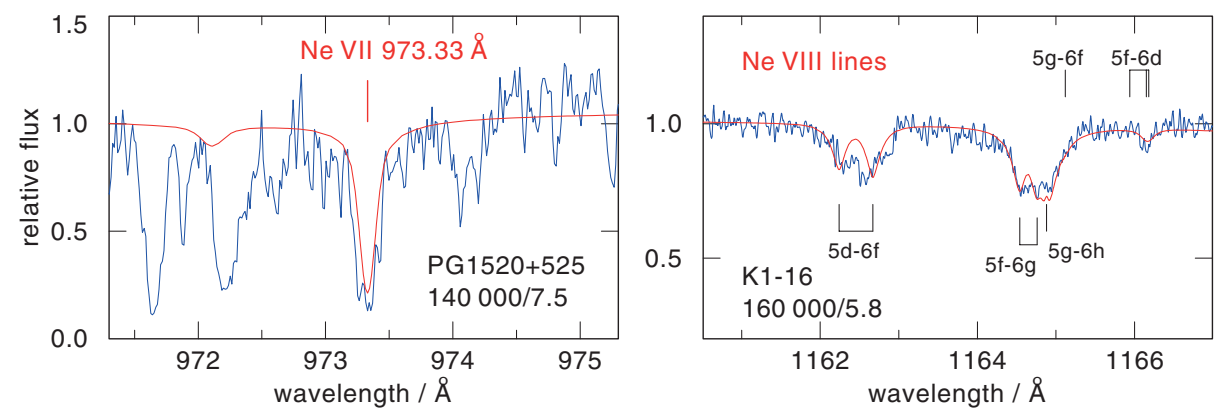

Figure 4. Highly ionized neon lines in PG1159-type central stars. Left: Ne VII $\lambda 973.3 \AA$ in PG1520+525. Right: Ne VIII lines in K 1-16. The neon abundance in the overplotted models is $2 \%$ by mass.

identified for the first time. From silicon, which is usually detected through its Si IV resonance doublet, very high ionization stages were discovered (Si V-VII).

Most trace metal abundances in PG1159 stars are consistent with predictions from nucleosynthesis models. For example, extreme fluorine abundances were determined in some stars (up to 200 times solar), while argon is solar. After the iron abundance problem was solved, the largest remaining discrepancy is for sulfur. In four out of five stars, we found unexpectedly strong depletions down to about 0.1 times solar or less (e.g. Jahn et al. 2007). It is remarkable, that such a sulfur anomaly is also encountered at abundance analyses of PNe (Henry et al. 2006).

The nitrogen abundance is an important marker for the event leading to hydrogendeficiency in PG1159 and [WC] stars. The absence (less than $0.1 \%$, by mass) or presence (few $\%$ ) of $\mathrm{N}$ is a reliable indicator of a late or very late thermal pulse (i.e. final pulse occurred in H-burning post-AGB state or WD cooling phase, respectively).

Neon deserves special attention. Its observed abundance is $2 \%$ (i.e. $\approx 15$ times solar), in agreement with expectations from stellar models. During evolution, all CNO is mostly transformed into ${ }^{14} \mathrm{~N}$, which is subsequently transformed to ${ }^{22} \mathrm{Ne}$ by $\alpha$ captures. The discovery of neon by the identification of Ne VII and Ne VIII in UV spectra and in the optical wavelength region led to the conclusion that previous identifications of ultrahighionization lines in WR stars (Pop. I and II) and in PG1159 stars must be revised.

\subsection{Neon vs. oxygen: wrong identification of $[W C]$ spectral classification lines}

In order to classify the hottest [WR] stars, in particular the [WO] (which are a continuation of $[\mathrm{WC}]$ to higher effective temperatures), line ratios of $\mathrm{O}$ VI/O viI/O vIII are used 
Table 1. Old ultrahigh ionization line identifications and revised identifications.

\begin{tabular}{|c|c|c|c|}
\hline Wavelength $(\AA)$ & Ultrahigh-ionization identification & New identif & ication \\
\hline 1932 & O VIII $n=7 \rightarrow 8$ & Ne VIII & $n=7 \rightarrow 8$ \\
\hline 2977 & O VIII $n=6 \rightarrow 7$ & Ne viII & $n=6 \rightarrow 7$ \\
\hline 3893 & O VII $\quad n=7 \rightarrow 8$ & $\begin{array}{l}\text { Ne VII } \\
\text { plus Ne VII }\end{array}$ & $\begin{array}{l}3 \mathrm{p}^{3} \mathrm{P}^{\mathrm{o}} \rightarrow 3 \mathrm{~d}^{3} \mathrm{D} \\
n=7 \rightarrow 8\end{array}$ \\
\hline 4340 & O VIII $n=8 \rightarrow 9$ & Ne VIII & $n=8 \rightarrow 9$ \\
\hline 4500 & $\mathrm{C}_{\mathrm{VI}} \quad n=8 \rightarrow 10$ & O vi & $n=8 \rightarrow 10$ \\
\hline 4555 & $n=9 \rightarrow 11$ & Ne VII & $n=9 \rightarrow 11$ \\
\hline 4945 & $n=6 \rightarrow 7$ & $\mathrm{NV}$ & $n=6 \rightarrow 7$ \\
\hline 5290 & $n=7 \rightarrow 8$ & O VI & $n=7 \rightarrow 8$ \\
\hline 5665 & O VII $n=8 \rightarrow 9$ & Ne VII & $n=8 \rightarrow 9$ \\
\hline 6068 & O VIII $n=9 \rightarrow 10$ & Ne viII & $n=9 \rightarrow 10$ \\
\hline
\end{tabular}

(Acker \& Neiner 2003). Lines from O viI and O viII are called "ultrahigh ionization" lines (Barlow et al. 1980), because temperatures of the order $10^{6} \mathrm{~K}$ are required to excite the respective energy levels. These are, however, not attained in the atmospheres unless e.g. shocks are invoked.

We have shown that the O VII and O VIII line identifications are probably wrong (Werner et al. 2007). All features can be ascribed to Ne VII and Ne VIII lines, respectively. This identification is much more natural because the neon lines are excited ordinarily in hot star atmospheres without invoking shocks, because and neon is an abundant element in [WC] and PG1159 stars. As a consequence, the putative O vi/O vir line ratios for example are in fact ratios of O VI to Ne VII lines. The current spectral classification scheme is therefore affected by the $\mathrm{O} / \mathrm{Ne}$ abundance in the star and not only by the temperature.

Other features that are commonly ascribed to ultrahigh ionization lines from carbon ( $\mathrm{C}$ V and $\mathrm{C}$ VI) are in fact lines from ordinarily excited $\mathrm{N}$ V and $\mathrm{O}$ VI, respectively. Table 1 summarizes the new line identifications.

\section{Two distinct post-AGB evolutionary sequences for H-deficient stars?}

The $\mathrm{O}(\mathrm{He})$ stars are a small group of four objects (two have an associated PN) with parameters close to $T_{\text {eff }}=120000 \mathrm{~K}$ and $\log g=6$ (Rauch et al. 1998, 2008, and Reindl et al. in these proceedings; Fig. 1). They have helium-dominated atmospheres and cannot be explained by a late flash like the PG1159 and [WC] stars, because evolutionary models always predict high $\mathrm{C}$ abundances. One can therefore argue that they are representatives of a distinct post-AGB sequence. If so, which stars are possible progenitors and successors?

It could be that some of the $\mathrm{O}(\mathrm{He})$ stars are evolved $\mathrm{R}$ CrB stars $\left(T_{\text {eff }}\right.$ around $\left.7000 \mathrm{~K}\right)$ and we are currently investigating how metal abundance patterns compare (see Reindl et al. in these proceedings). Even if the relationship is identified, the question about their origin remains unanswered. $\mathrm{R}$ CrB stars may be the outcome of a double-degenerate merger, i.e. the coalescence of a carbon-oxygen WD with a helium WD. Merger simulations indicate that the resulting chemical abundance patterns are in qualitative agreement with observed abundances in $\mathrm{R} \mathrm{CrB}$ stars, although it is not clear to what extent the resulting metal abundances are determined by the intershell composition of the C-O WD progenitor or by nucleosynthesis during merging (Jeffery et al. 2011, Longland et al. 2011). Helium-rich objects that bridge the large $T_{\text {eff }}$ gap between the $\mathrm{R} \mathrm{CrB}$ and $\mathrm{O}(\mathrm{He})$ 
stars could be the extreme He-B stars $\left(\mathrm{EHeB}, T_{\text {eff }}\right.$ around $\left.20000 \mathrm{~K}\right)$ and a handful known low-gravity sdO stars ( $T_{\text {eff }}$ around $70000 \mathrm{~K}$, see e.g. Jeffery 2008).

Two of the $\mathrm{O}(\mathrm{He})$ stars have a significant amount of hydrogen $(\mathrm{H} / \mathrm{He}=0.1$ and 0.5 by number in HS 1522+6615 and LoTr 4, respectively; Rauch et al. 1998; Reindl et al. in these proceedings). Also, a few R CrB stars have much hydrogen (e.g. $\mathrm{H} / \mathrm{He}=0.5$ in V854 Cen; Rao \& Lambert 1996), as well as some EHeB stars. This is in conflict with the merger scenario because one would expect very little or no remaining hydrogen. It is speculated that an alternative origin are post-early AGB stars, i.e. rather low-mass objects that experience their first thermal pulse after departure from the AGB (Miller Bertolami \& Althaus 2006).

Immediate progenitors of the $\mathrm{O}(\mathrm{He})$ stars might be [WN] central stars. Their existence, however, is still debated (Todt et al. 2010a). It is claimed that the central star of PB 8 $\left(T_{\text {eff }} \approx 50000 \mathrm{~K}\right)$ is indeed a [WN] (or more precise: a [WN6]/[WC7] type), being Hedominated with a large $\mathrm{H}$ abundance $(\mathrm{H} / \mathrm{He} / \mathrm{C} / \mathrm{N} / \mathrm{O}=40 / 55 / 1.3 / 2 / 1.3$, mass fractions $)$, and a possible relation to the $\mathrm{O}(\mathrm{He})$ class was discussed (Todt et al. 2010b). Miller Bertolami et al. (2011) argue that PB 8 might be the result of a diffusion-induced nova, which is an entirely different scenario to explain H-deficient post-AGB stars. It could perhaps also apply to the $\mathrm{O}(\mathrm{He})$ stars.

A potential successor of the $\mathrm{O}(\mathrm{He})$ stars could be the He-dominated KPD $0005+5106$ $\left(T_{\text {eff }}=200000 \mathrm{~K}, \log g=6.7\right.$; Wassermann et al. 2010). Its metal abundance pattern reveals strong commonalities with R CrB stars. Like the PG1159 stars, with increasing gravity the $\mathrm{O}(\mathrm{He})$ stars will evolve into DA or non-DA WDs depending on whether they retained hydrogen or not.

\section{Evidence for H-deficient post-super AGB evolution}

$\mathrm{H} 1504+65$ is the hottest known white dwarf $\left(T_{\text {eff }}=200000 \mathrm{~K}\right)$. Its high surface gravity $(\log g=8)$ indicates a relatively high mass. Spectroscopically, it is related to the PG1159 class but it is a distinct object because it is not only hydrogen-deficient but also heliumdeficient. The atmosphere is primarily composed of carbon and oxygen, by equal amounts Werner (1991). In addition, a high abundance of neon was derived Werner \& Wolff (1999). The origin of this exotic surface chemistry $(\mathrm{C}=49 \%, \mathrm{O}=49 \%, \mathrm{Ne}=2 \%$, mass fractions $)$ is completely unclear. We have speculated that $\mathrm{H} 1504+65$ represents the naked $\mathrm{C}-\mathrm{O}$ core of a white dwarf. Another, even more exciting possibility is that we see the eroded $\mathrm{C}-\mathrm{O}$ envelope of a $\mathrm{O}-\mathrm{Ne}-\mathrm{Mg}$ white dwarf. This is corroborated by a Chandra soft Xray spectrum (Werner et al. 2004) that allowed the detection of magnesium, with an abundance of about $2 \%$ (Fig. 5). Another strong argument in favor of this idea would be the detection of sodium, which would be direct evidence for C-burning. Stellar models predict that the ${ }^{23} \mathrm{Na}$ abundance at the bottom of the $\mathrm{C} / \mathrm{O}$ envelope is comparable to that of neon (main isotope $\left.{ }^{20} \mathrm{Ne}\right)$ and magnesium $\left({ }^{24,25,26} \mathrm{Mg}\right.$, Iben et al. 1997). We are analyzing new UV spectra taken with HST/COS aiming at the abundance determinations for $\mathrm{Mg}$ and $\mathrm{Na}$ (Werner et al. 2010).

It could turn out that $\mathrm{H} 1504+65$ is one of the "heavy-weight" intermediate-mass stars $\left(8 \mathrm{M}_{\odot} \lesssim M \lesssim 10 \mathrm{M}_{\odot}\right)$ that form white dwarfs with electron-degenerate $\mathrm{O}-\mathrm{Ne}-\mathrm{Mg}$ cores. At present it is uncertain under which circumstances super-AGB stars (i.e. the massive counterparts of AGB stars that ignite carbon but do not proceed to further stages of nuclear burning) produce $\mathrm{O}-\mathrm{Ne}-\mathrm{Mg}$ WDs or explode as electron-capture SNe producing NSs (e.g. Siess 2007). This uncertainty mainly arises from modeling uncertainties in mass-loss and mixing processes. The possibility that $\mathrm{H} 1504+65$ is a $\mathrm{O}-\mathrm{Ne}-\mathrm{Mg}$ $\mathrm{WD}$ is remarkable, because evidence for the existence of such objects is rather scarce 


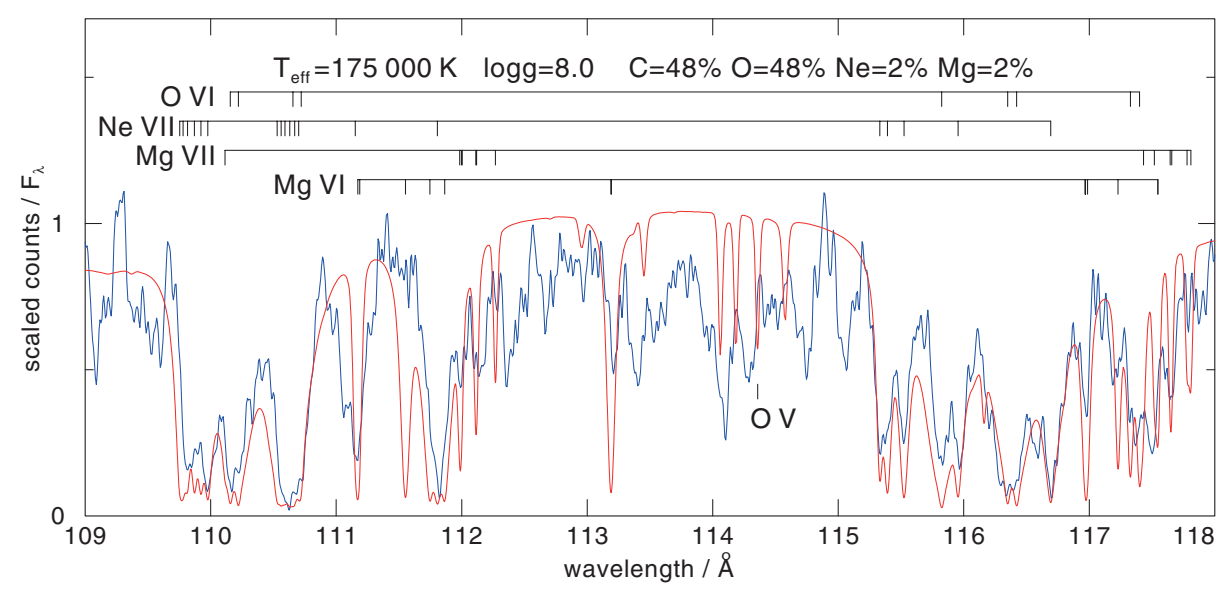

Figure 5. Detail from a Chandra soft X-ray spectrum of H $1504+65$ and an overplotted model with parameters as noted in the panel.

(Weidemann 2003). Evidence from single massive WDs is weak, and the most convincing cases are WDs in binary systems. Strong Ne overabundances are found in novae (Livio \& Truran 1994) or in eroded WD cores in LMXBs (Juett et al. 2001). If a high Na abundance is found in $\mathrm{H} 1504+65$, then this would be the most compelling case for the existence of a single O-Ne-Mg WD, i.e. a post super-AGB star. H 1504+65 would then also challenge stellar evolution theory relevant for super-AGB stars, because it cannot explain how the star has lost its H-rich and He-rich envelopes and why it exposes its metallic core.

At any rate, one may wonder where potential progenitors and successors with H1504like chemistry are. All [WR] and PG1159 stars analyzed so far are still having significant amounts of helium in their atmospheres (30-50\%) and always $\mathrm{C} \gg \mathrm{O}$. Recently, a group of relatively cool white dwarfs $\left(T_{\text {eff }} \approx 20000 \mathrm{~K}\right)$ with almost pure carbon atmospheres were discovered (so-called "hot DQs"; Dufour et al. 2007, 2011). They may be evolved H1504-like stars because one can expect that at some point in future evolution carbon as lightest element will float on top of $\mathrm{H} 1504+65$.

Recently, Gänsicke et al. (2010) discovered a new class of two O-rich white dwarfs (He-dominated atmospheres with $\mathrm{O} / \mathrm{He} \approx 0.01$ and $\mathrm{O}>\mathrm{C}$, by number, and $T_{\text {eff }}$ around $10000 \mathrm{~K}$ ) that could be $\mathrm{O}-\mathrm{Ne}-\mathrm{Mg}$ white dwarfs.

\section{Summary and conclusion}

The rich diversity of hydrogen-deficient central stars of planetary nebulae and related objects is not well understood. The majority consists of [WC] and PG1159 stars which are thought to form an evolutionary sequence that is caused by a late helium-shell flash in post-AGB stars. The helium-dominated $\mathrm{O}(\mathrm{He})$ stars suggest that there exists at least one additional H-deficient sequence that is caused by a double-degenerate merger. It is possible that the $\mathrm{O}(\mathrm{He})$ stars are the progeny of $\mathrm{R}$ CrB stars.

There is growing evidence that there exists a H-deficient post-super AGB white-dwarf cooling sequence consisting of remnants with $\mathrm{O}-\mathrm{Ne}-\mathrm{Mg}$ cores. $\mathrm{H} 1504+65$ with its $\mathrm{C}$ $\mathrm{O}$ dominated atmosphere might mark the hot end of this sequence while the recently discovered groups of C-dominated "hot DQs" and He-dominated O-rich white dwarfs occupy cooler regions. The origin of H-deficiency in post-super AGB stars is unknown. There is no obvious hint as to which central stars of planetary nebulae could be related to these chemically peculiar white dwarfs. 


\section{Acknowledgements}

I thank Thomas Rauch and Jeff Kruk for their enduring collaboration. HST data analysis in Tübingen is supported by the German Ministry of Education and Research through the German Aerospace Center (grant 05 OR 0806).

\section{References}

Acker, A. \& Neiner, C. 2003, A\&A, 403, 659

Althaus, L. G., Panei, J. A., Miller Bertolami, M. M., García-Berro, E., Córsico, A. H., Romero, A. D., Kepler, S. O., \& Rohrmann, R. D. 2009, ApJ, 704, 1605

Barlow, M. J., Blades, J. C., \& Hummer, D. G. 1980, ApJ (Letters), 241, L27

Crowther, P. A. 2008, in: K. Werner \& T. Rauch (eds.), Hydrogen-Deficient Stars, ASP-CS (ASP: San Francisco), 391, 83

Crowther, P. A., De Marco, O., \& Barlow, M. J. 1998, MNRAS, 296, 367

Dufour, P., Liebert, J., Fontaine, G., \& Behara, N. 2007, Nature, 450, 522

Dufour, P., Béland, S., Fontaine, G., Chayer, P., \& Bergeron, P. 2011, ApJ (Letters), 733, L19

Gänsicke, B. T., Koester, D., Girven, J., Marsh, T. R., \& Steeghs, D. 2010, Science, 327, 188

Henry, R. B. C., Skinner, J. N., Kwitter, K. B., \& Milingo, J. B. 2006, in: M. J. Barlow \& R.H. Méndez (eds.), Planetary Nebulae in our Galaxy and Beyond, Proc. IAU Symp. No. 235 (Cambridge: Cambridge University Press), p. 417

Iben, I. Jr., Ritossa, C., \& García-Berro, E. 1997, ApJ, 489, 772

Jahn, D., Rauch, T., Reiff, E., Werner, K., Kruk, J. W., \& Herwig, F. 2007, A\&SA, 462, 281

Jeffery, C. S. 2008, in: K. Werner \& T. Rauch (eds.), Hydrogen-Deficient Stars, ASP-CS (ASP: San Francisco), 391, 3

Jeffery, C. S., Karakas, A. I., \& Saio, H. 2011, MNRAS, 414, 3599

Juett, A. M., Psaltis, D., \& Chakrabarty, D. 2001, ApJ, 560, L59

Keller, G. R., Herald, J. E., Bianchi, L., Maciel, W. J., \& Bohlin, R. C. 2011, MNRAS, 418, 705

Livio, M. \& Truran, J. W. 1994, ApJ, 425, 797

Longland, R., Lorén-Aguliar, P., José, J., García-Berro, E., Althaus, L. G., \& Isern, J. 2011, $A p J$ (Letters), 737, L34

Méndez, R. H., Miguel, C. H., Heber, U., \& Kudritzki, R. P. 1986, in: K. Hunger, D. Schönberner, \& N. K. Rao (eds.), Hydrogen Deficient Stars and Related Objects, Proc. IAU Coll. No. 87 (Reidel: Dordrecht), p. 323

Miller Bertolami, M. M. \& Althaus, L. G. 2006, A\& $A, 454,845$

Miller Bertolami, M. M., Althaus, L. G., Olano, C., \& Jiménez, N. 2011, MNRAS, 415, 1396

Rao, N. K. \& Lambert, D. L. 1996, in: C. S. Jeffery \& U. Heber (eds.), Hydrogen-Deficient Stars, ASP-CS (ASP: San Francisco), 96, 43

Rauch, T., Dreizler, S., \& Wolff, B. 1998, A\& A, 338, 651

Rauch, T., Reiff, E., Werner, K., \& Kruk, J. W. in: K. Werner \& T. Rauch (eds.), HydrogenDeficient Stars, ASP-CS (ASP: San Francisco), 391, 135

Siess, L. 2007, A\&A, 476, 893

Todt, H., Peña, M., Hamann, W.-R., \& Gräfener, G. 2010a, in: K. Werner \& T. Rauch (eds.), 17th European White Dwarf Workshop, AIP-CP (Melville: New York), 1273, 219

Todt, H., Peña, M., Hamann, W.-R., \& Gräfener, G. 2010b, A\&A, 515, A83

Wassermann, D., Werner, K., Rauch, T., \& Kruk, J. W. 2010, A\&A, 524, A9

Weidemann, V. 2003, in D. de Martino, R. Silvotti, J.-E. Solheim \& R. Kalytis (eds.), White Dwarfs, NATO Sci. Ser. II (Kluwer: Dordrecht), 105, 3

Werner, K. 1991, AछA, 251, 147

Werner, K. \& Wolff, B. 1999, A\&̊A, 347, L13

Werner, K. \& Herwig, F. 2006, PASP, 118, 183

Werner, K., Rauch, T., Barstow, M. A., \& Kruk, J. W. 2004, A\&A, 421, 1169

Werner, K., Rauch, T., \& Kruk, J. W. 2007, A\&A, 466, 317

Werner, K., Rauch, T., Reiff, E., \& Kruk, J. W. 2008, in: K. Werner \& T. Rauch (eds.), HydrogenDeficient Stars, ASP-CS (ASP: San Francisco), 391, 109

Werner, K., Rauch, T., \& Kruk, J. W. 2010, ApJ (Letters), 719, L32

Werner, K., Rauch, T., Kruk, J. W., \& Kurucz, R. L. 2011, A\&A, 531, A146 